\title{
Emergency hernia surgery during COVID-19 outbreak: delayed presentations, more resections
}

\author{
Charalampos Seretis ${ }^{1,2}$ (D) \\ 1 Department of General Surgery, George Eliot Hospital NHS Trust, Nuneaton, United Kingdom \\ ${ }^{2}$ Medical School, University of Warwick, United Kingdom
}

Cite this article as: Seretis C. Emergency hernia surgery during COVID-19 outbreak: delayed presentations, more resections. Turk J Surg 2021; 37 (2): 197-198.

Corresponding Author

Charalampos Seretis

E-mail: babismed@gmail.com

Received: 06.03 .2021

Accepted: 19.04.2021

Available Online Date: 30.06 .2021

() Copyright 2021 by Turkish Surgical Society Available online at www.turkjsurg.com

DOI: $10.47717 /$ turkjsurg.2021.5267
Dear Editor,

The outbreak of COVID-19 pandemic has, without a doubt, affected the provision of emergency and elective surgery on a worldwide scale. In contrast to elective procedures, emergency surgery can hardly be postponed due to the associated increased morbidity and mortality risks; hence the public has been globally encouraged to seek medical attention upon the development of acute symptoms $(1,2)$. However, in our anecdotal experience, and being in accordance to recently published data, there has been a drop in the volume of the emergency hospital admissions, with a notable increase of the complexity of the presenting problems (3). With respect to surgery, and in particular emergency hernia surgery, we have noticed a trend of delayed admission from the onset of symptoms, along with an increased percentage of non-salvageable visceral contents during emergency hernia repairs. We have therefore reviewed our operative records of emergency hernia repairs since the onset of the pandemic in our catchment area (Warwickshire, United Kingdom) between 03/2020-02/2021 (COVID-19 pandemic year) and compared them with the relevant data from 03/2019-02/2020 (pre-COVID-19 pandemic year). Of note, our hospital (district general) has been providing continuously uninterrupted emergency general surgery services in our region during the COVID-19 pandemic outbreak. However, between 03-05/2020 and 01-02/2021 there was a substantial reduction in the volume of elective operations (including hernias), with prioritization of clinically urgent cases, such as surgical oncology operations, as sole elective procedures, due to increased hospital pressures from the pandemic.

Reviewing our records for the 2020-2021 study period, we performed 46 emergency hernia operations, while 29 cases were operated between the respective 20192020 observation period; therefore, resulting in a more than $50 \%$ increase of our emergency hernia workload during the pandemic. Interestingly, the average time from onset of symptoms of incarceration/strangulation to the eventual assessment by the on-call surgical team increased in the COVID-19 period to 2.7 days from 1.2 days in the pre-COVID-19 year. The latter resulted in a significant increase of the need for intestinal resections due to non-viability of the herniated viscera, from $10.3 \%$ (2/29 cases) in 2019-2020 to $17.4 \%$ (8/46 cases) in 2020-2021. Although there was no major postoperative morbidity or mortality in our case series, either in 2019-2020 or 2020-2021 (only Clavien-Dindo grade II/III -wound infections, superficial post-operative collections, urinary retentions / no occurrence of anastomotic leaks, re-operations or unplanned returns to the Intensive Care Unit), there was a significant increase of the average length of stay of the patients operated during the 2020-2021 period (5.77 days in 2020-2021 vs 3.01 days in 2019-2020), to an extent expected due to the higher resection rates during the pandemic year. With 
respect to possible hospital-acquired COVID-19 infection, none of our patients was tested positive (nose-throat swab for PCR) during any stage of their hospitalization, and none developed any major respiratory tract complication.

Based on our observations as a district general hospital emergency surgical service, in light of the recurrent relapses of COVID-19 infection waves in the community -and until the immunisation programmes cover the majority of the general population- we strongly advocate that patients with acutely symptomatic hernias should be encouraged to seek surgical specialist input with no delays. In our experience since the outbreak of the pandemic, emergency hernia surgery can be safely performed with very low risk for respiratory complications and postoperative care can be delivered without unplanned intensive care admissions. We would reserve taxis of the acutely symptomatic hernias only for patients who are unfit or unwilling for surgery.

\section{REFERENCES}

1. Hussain PM, Kanwal A, Gopikrishna D. Resuming elective operations after COVID-19 pandemic. Br J Surg 2020; 107(11): e549. [CrossRef]

2. Poeran J, Zhong H, Wilson L, Liu J, Memtsoudis SG. Cancellation of elective surgery and intensive care unit capacity in New York State: a retrospective cohort analysis. Anesth Analg 2020; 131(5): 1337-41. [CrossRef]

3. Cano-Valderrama O, Morales X, Ferrigni CJ, Martín-Antona E, Turrado $V$, García A, et al. Acute care surgery during the COVID-19 pandemic in Spain: changes in volume, causes and complications. A multicentre retrospective cohort study. Int J Surg 2020; 80: 157-61. [CrossRef] 